\title{
2-D velocity field structure and oscillations nearby quiescent filament
}

\author{
G. P. Mashnich ${ }^{1}$ †, V. S. Bashkirtsev ${ }^{1}$ E. M. Golubeva ${ }^{1}$ A. I. \\ Khlystova $^{1}$ and A. S. Letunov ${ }^{1}$ \\ ${ }^{1}$ Institute of Solar-Terrestrial Physics, P.O. Box 4026, Irkutsk, Russia \\ email: mashnich@iszf.irk.ru
}

\begin{abstract}
We have developed the technique and software to observe and process spectral data obtained with the CCD camera. It makes possible to construct two-dimensional maps of Doppler velocity and intensity in solar features simultaneously at two levels in the solar atmosphere. We present some results derived from study of the structure and space-time variations of the Doppler velocity in the region of quiescent filaments in the chromosphere (the $H_{\beta} \lambda 486.1 \mathrm{~nm}$ line) and under filaments in the photosphere (the Fe I $\lambda 486.3 \mathrm{~nm}$. and Fe I $\lambda 485.9 \mathrm{~nm}$ lines). We came to a conclusion that motions in quiescent filaments consist of both the stationary and oscillatory components and follow helical trajectories.
\end{abstract}

\section{Introduction}

At present time the following filament properties have been established: in filaments mass flows are oriented by the magnetic field (see e.g. Bashkirtsev \& Mashnich 1980; Hood \& Anzer 1990), in the filament bodies the structure directions can be either dextral or sinistral Martin (1998). Flows of opposite directions were detected in neighboring elements inside of quiescent filaments and were called by Zirker et al. (1998) as counterstreaming flows. The discovery of the photospheric motions under filaments converging to a neutral line (see e.g. Litvinenko \& Martin 1999) were followed by the theoretical models, showed how the converging photospheric motions produce magnetic field configurations which are able to sustain the dense prominence plasma in a hot corona. The interaction of photospheric motions with magnetic fields take place in the whole solar atmosphere, and it is important to identify types of motions which affect the prominence evolution. In this report we present observational results of the structure and dynamics of the velocity field in and near by the quiescent filament during its passage over the solar disk.

\section{Data Processing, Observation Results and Concluding Remarks}

Between July 4 and July 8, 2003 the spectral observations of a large filament were carried out with the horizontal solar telescope at Sayan Solar Observatory. The additional device with the birefringent $H_{\alpha}$-filter is used to select the study targets and to control their positions on the mirror slit of spectrograph at the time of observation. The filament parts under consideration are scanned on the spectrograph slit with the 2 arcsec spatial step, so that a spectrum at every given position is taken. The CCD camera of the $1024 \times 256$ pixels is placed in the cassette part of the spectrograph. The spectrum height is 256 pixels along the spectrograph slit and corresponds to 65 arcsec on the solar disk. The Doppler shift values are determined as shifts of spectral line at a given position in

$\dagger$ Present address: Institute of Solar-Terrestrial Physics, Lermontova, 126, Irkutsk, Russia 


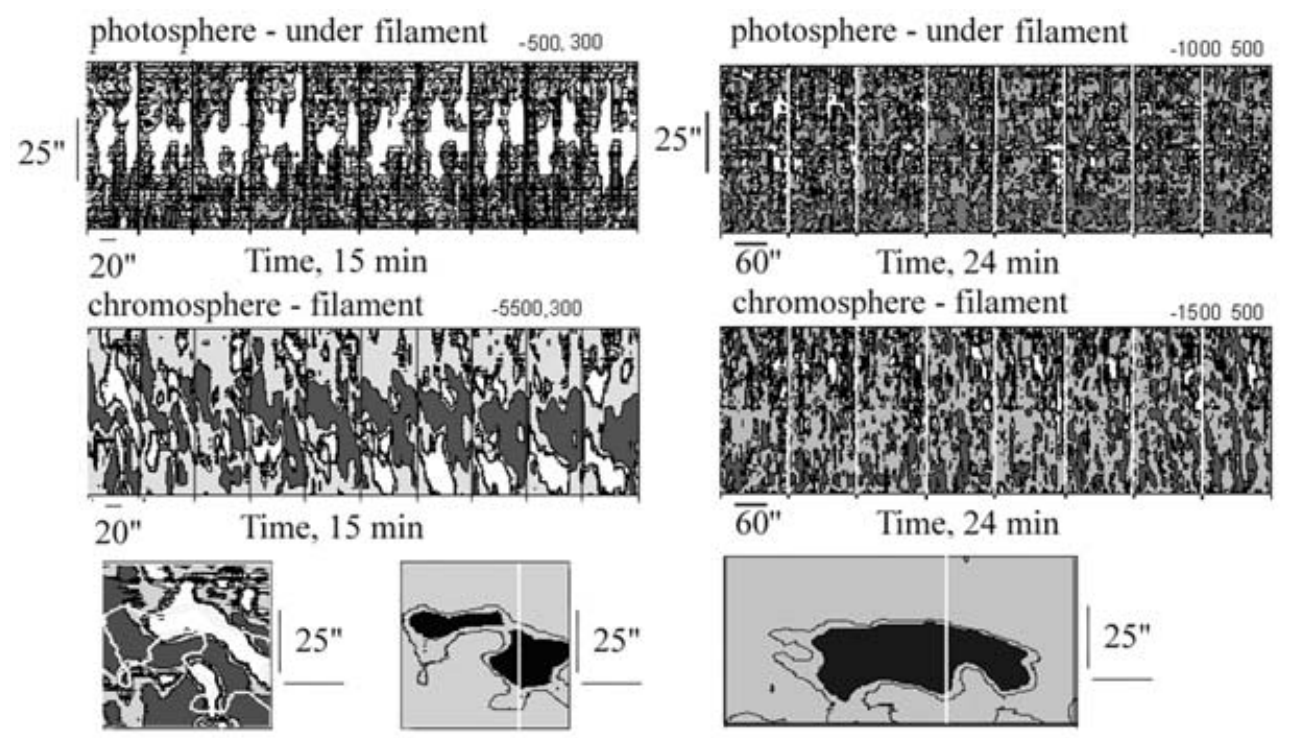

Figure 1. The sequences of the Doppler velocity maps in the photosphere (top) under the filament and in the filament (in the middle): the left panel - July 5, 2003, and the right panel July 8,2003 . The boundaries between scans are pointed by vertical dividing lines. The motions towards the observer are presented by dark gray color, while the white color shows motions in the opposite direction. The first map in chromosphere and general view of the filament fragments in $H_{\beta}$ intensity are disposed at the bottom. The vertical lines on the intensitogramms point to the position where Doppler velocity temporal variations were registered.
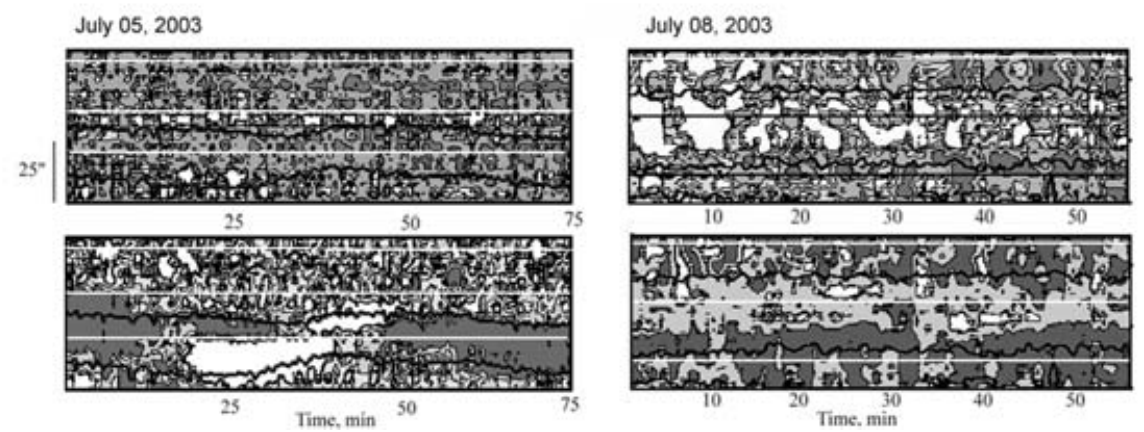

Figure 2. The Velocity variations in the filaments. Fragments of the recordings (about $180 \mathrm{~min}$ with cadence of $30 \mathrm{sec}$ ) July 5, 2003 in the photosphere (top) and in the chromosphere (bottom) at the left. At the right - on July 8, 2003. Fragments of the recordings (about 180 minutes with a cadence of $30 \mathrm{sec}$ ) in the photosphere (top) and in the chromosphere (bottom).

reference to the averaged spectrum which is used as a spectrum with zero shift. In the photosphere (the lines of Fe I $\lambda 486.3 \mathrm{~nm}$ and Fe I $\lambda 485.9 \mathrm{~nm}$ ) and chromosphere (the line of $H_{\beta} \lambda 486.1 \mathrm{~nm}$ ) the Doppler velocities are obtained simultaneously in this way, and two-dimensional maps of both intensity and velocity are constructed. The solar full disk $H_{\alpha}$-images (from Internet) are used to determine the angle between the line-of-sight and the long axis of the filament.

On July 5, 2003 the filament was situated at E40N17. The angle $\theta$ between the long filament axis and the line-of-sight was small. The large regions of the opposite directed motions were found out on the different sides from of the long filament axis. Such structures of Doppler motions inside filaments often take place when the angle between the 

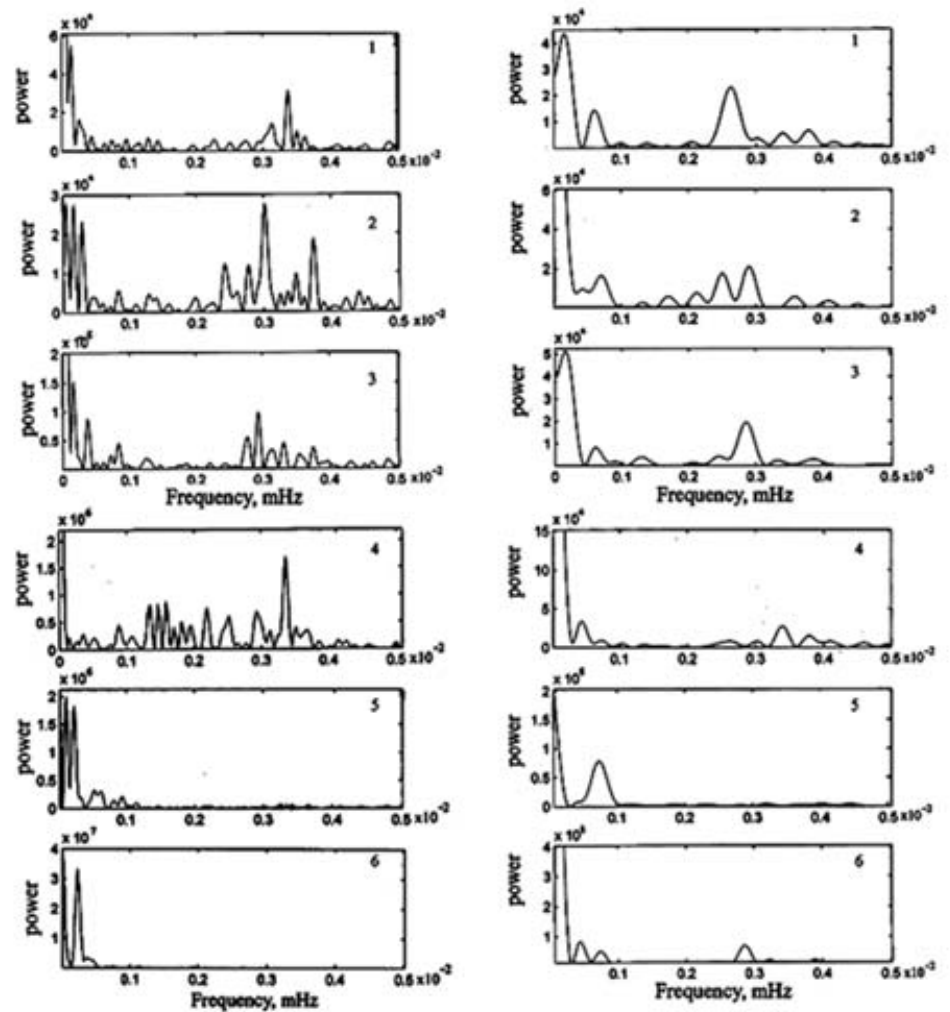

Figure 3. The photospheric and chromospheric the power spectra of the oscillations for positions marked on the velocity variation maps by the horizontal line. There are at the left panel on July 5, 2003 1-photosphere, 2-photosphere under filament edge, 3-under filament, 4-chromosphere, 5-filament edge, 6-filament. At the right panel spectra of the oscillations on July 8, 2003: 1-photosphere, 2- photosphere under filament, 3-under filament edge, 4-chromosphere, 5-filament, 6-filament edge.
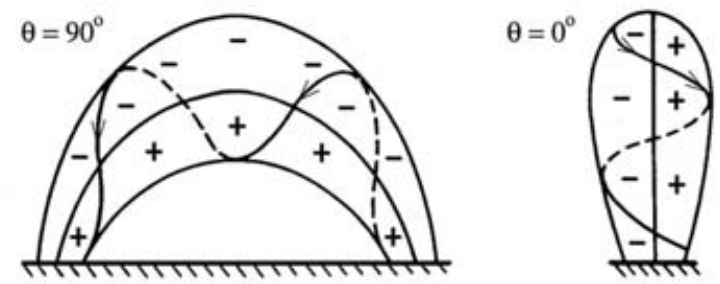

Figure 4. The representation of the motions in the quiescent filament at values $\theta=90^{\circ}$ and $\theta=0^{\circ}$. The minus are the Doppler velocities toward observer and plus are the opposite direction.

long filament axis and the line-of-sight are small enough (Mashnich et al. (2002)). In Figure 1 (the left panel) the Doppler velocity maps are presented one by one to make the temporal sequence of scans. The Figure 1 illustrates clearly the transverse motions in filament as systematic displacements of the Doppler velocity structures. The transverse velocity component is about $4.5 \mathrm{~km} / \mathrm{s}$. The fragments of the Doppler velocity variation recordings (Figure 2) show that the motions in the photosphere under the filament and in the filament consist of systematic flows and oscillating components. One can see that 
the filament edges are located above the boundaries of the opposite directed photosphere motions. The boundaries under the filament have displaced during the observation. In the filament the Doppler motions have the oscillating character and demonstrate backand-forth motions along the filament axis. The filament oscillation modes differ from the chromosphere oscillations on the spatial scales and on periods. It is necessary to note that outside the filament the photospheric and chromospheric Doppler motions are differently directed to each other. On July 8, 2003 the filament crossed the central meridian. The Figure 1 (see the right panel) demonstrates the sequences of the photospheric and chromospheric Doppler velocity maps for that day. In this case the angle $\theta$ between the filament axis and line-of-sight is about $90^{\circ}$. One can recognize that photospheric plasma rise toward lateral filament feet and keep the same direction inside the filament, until it go down on the other side of the filament. On the right panel Figure 2 are presented the photospheric and chromospheric Doppler velocity oscillations for this day. The distributions and displacements of the Doppler velocity structures observed in the filament most likely can be explained as the oscillating motion of material along spiral trajectories. Results of the spectral analysis of the Doppler velocity oscillations inside the filament, at its edge and outside are illustrated in Figure 3. As seen from the Figure (the left panel), on July 5, 2003, the following spatial distribution takes place. The chromosphere the quasi-hourly periods (63 minutes) are the most powerful in the filament. Oscillating periods of 20-60 minutes are detected at the edge of the filament. Outside the filament the velocity fluctuation spectra are typical for the chromosphere. Here the photospheric spectra at the shot-period range has the similar character. Both long-period oscillations and shot-period ones are observed under the filament and in the photosphere. Close to the central meridian (see the right panel in Figure 3) the character of spectra change. The period of $20 \mathrm{~min}$ is appeared in the filament spectrum. The amplitudes of the chromospheric spectra come to one order of magnitude smaller as well as the photospheric spectra under the filament. The observational results could be explained by the general scheme of filament motions in Figure 4. Our earlier investigations Bashkirtsev \& Mashnich (1984) of the oscillatory processes in prominences are consistent with results of this work.

The findings of our investigation are of some interest primarily because the line-of-sight velocity measurements were made simultaneously at two levels in the solar atmosphere. Our studies of the time variations and two-dimensional spatial distribution of the velocities allow us to infer that the matter in quiescent filaments moves along the spiral trajectories. In the filament the oscillating modes differ from the chromosphere oscillations in spatial scale and in period. The downward mass flows are often observed in the photosphere under the filament. The measured velocities contain both the stationary and oscillatory components. This work was supported by the Federal program 'Astronomy' 1105 .

\section{References}

Bashkirtsev, V.S. \& Mashnich, G. P. 1980 Phys. Solariterrestris 13, 118-122.

Bashkirtsev, V. S., \& Mashnich, G. P 1984 Sol. Phys. 91, 93-101.

Hood, A.W. \& Anzer, U. 1990 Sol. Phys. 126, 117-126.

Litvinenko, Yu. \& Martin, S. 1999 Sol. Phys. 190, 45-58.

Martin, S.F. 1998 Sol. Phys. 182, 107-137.

Mashnich, G.P., Bashkirtsev, V.S., Golubeva, H.M., \& Khlystova, A.I 2002 In Tenth European Solar Physics Meeting Solar variability: from core to outer frontiers (ed. A. Wilson), vol. 506, pp. 457-460. ESA.

Zirker, J.B., Engvold, O., \& Martin, S. 1998 Nature 396, 440-443. 